\title{
ELECTROTHERMAL SCS MICROMIRROR WITH LARGE-VERTICAL-DISPLACEMENT ACTUATION
}

\author{
Ankur Jain, Hongwei Qu, and Shane Todd \\ Department of Electrical \& Computer Engineering \\ University of Florida, Gainesville, FL 32611-6200
}

\author{
Gary K. Fedder \\ Department of Electrical \& Computer Engineering \\ Carnegie Mellon University, Pittsburgh, PA 15213
}

\section{ABSTRACT}

This paper reports a novel large-vertical-displacement (LVD) microactuator that can generate large piston motion at low driving voltage. A LVD micromirror device has been fabricated by using a unique DRIE CMOS-MEMS process that can simultaneously provide thin-film and single-crystal silicon microstructures. With only a $0.7 \mathrm{~mm}$ by $0.32 \mathrm{~mm}$ sized device, the LVD micromirror demonstrated a vertical displacement of $0.2 \mathrm{~mm}$ at an actuation voltage of $6 \mathrm{~V}$ d.c. This device can also perform bidirectional rotational scanning through the use of two bimorph actuators. The micromirror can rotate $\pm 20^{\circ}$, and has negligible initial tilt angle and high scanning speeds $(\sim 2 \mathrm{kHz})$.

\section{INTRODUCTION}

Fast-scanning mirrors with piston-motion are required by applications for wave-front shaping in adaptive optics [1], biomedical imaging, interferometry systems [2], laser beam scanning, and by spatial light modulators [3]. Other applications that require large vertical displacements include scanning tunneling microscopes, tunable lenses for confocal microscopy [4], microvalves, actuators on magnetic recording heads, and precision micropositioning systems.

Numerous piston-motion actuation designs have been reported in literature. Vertical displacements of $7.5 \mu \mathrm{m}$ [5] and even as high as $50 \mu \mathrm{m}$ [4] have been achieved through the use of electrostatic vertical comb drives. Deformable mirrors with electromagnetic and electrostatic actuations for use in adaptive optics demonstrated vertical displacements of $20 \mu \mathrm{m}$ [6] and

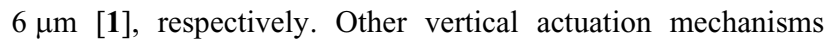
include a piezoelectric cantilever with displacements up to $25 \mu \mathrm{m}$ [7], and an electrothermally-actuated trampoline-type micromirror with a maximum displacement of $2 \mu \mathrm{m}$ [8]. Most of these actuators can only generate up to a few tens of microns of vertical displacement.

This paper presents a novel large-vertical-displacement (LVD) micromirror design that can perform optical scans, as well as large piston motion at low driving voltages. Out-ofplane displacement of the micromirror is provided by a pair of electrothermal actuators. It is well known that there is large $z$ displacement at the tip of a long rotational beam. The innovation of this LVD device is converting the large tip displacement into a pure $z$-axis displacement of a flat micromirror. The LVD microactuator design can potentially achieve maximum vertical displacements of a few millimeters with millimeter-sized devices.

In this paper, the LVD concept, device design and fabrication process will be described first. Then the experimental results, including frequency response, pistonmotion and bi-directional scanning, are reported.
Huikai Xie

Department of Electrical \& Computer Engineering

University of Florida, Gainesville, FL 32611-6200

\section{LVD MICROMIRROR DESIGN}

In prior work, we demonstrated single-crystal silicon (SCS) based 1-D and 2-D scanning micromirrors with large rotation angle for transverse scanning in optical coherence tomographic (OCT) imaging $[\mathbf{9 , 1 0}]$, but the unidirectional operation, nonstationary center of rotation, and large initial tilt angle of those micromirrors complicated the device packaging and optical design. The LVD micromirror consists of two complementary electrothermal actuators in a folded structure which allows the mirror plate to remain parallel to the substrate surface, while still taking advantage of the large stroke lengths provided by the actuators.

The schematic drawing of the LVD micromirror is illustrated in Fig. 1(a). The mirror plate is attached to a rigid silicon frame by a set of aluminum/silicon-dioxide bimorph beams. A polysilicon resistor is embedded within the silicon dioxide layer to form the heater for the thermal bimorph actuator. As this set of beams directly actuates the mirror, it is referred to as the mirror actuator. The movable frame is connected to the silicon substrate by another set of identical bimorph beams. This second set of beams actuates the frame, and is referred to as the frame actuator. After the mirror is released during fabrication, the beams curl up due to the tensile stress in the upper aluminum layer and compressive residual stress in the bottom silicon dioxide layer, as illustrated in Figs. 1(b) and 2. The rigidity of the frame and the flatness of the
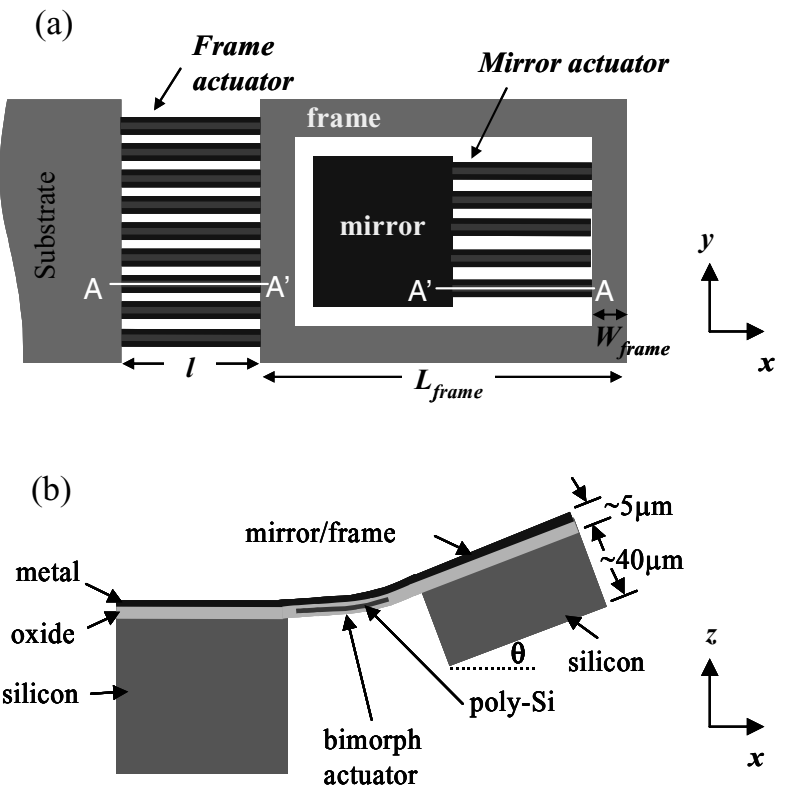

Figure 1.Design schematic of the LVD micromirror. (a) Topview; (b) crossectional view of $A-A^{\prime}$ '.

Travel support has been generously provided by the Transducers Research Foundation and by the DARPA MEMS and DARPA BioFlips programs. 

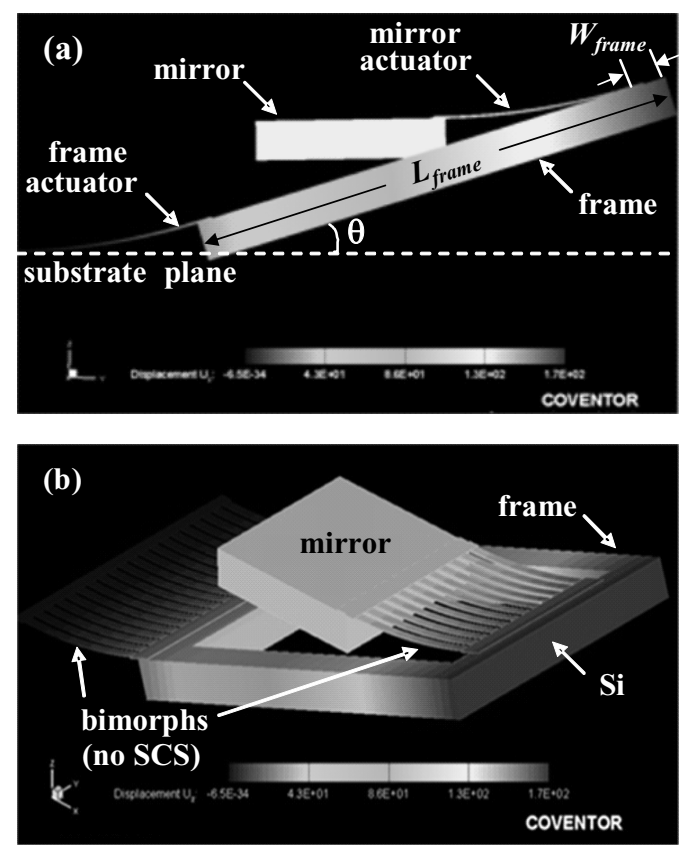

Figure 2. (a) Side-view; and (b) $3 D$ model of the LVD micromirror illustrating the initial curling of the bimorph actuators. The mirror surface is parallel to the substrate plane, i.e., no initial tilt, as the curling of the two bimorph actuators compensate each other. The mirror plate is located $0.13 \mathrm{~mm}$ above the substrate plane.

mirror are guaranteed by the thick SCS layer underneath the frame and mirror. In contrast, the bimorph actuators do not have the SCS layer and thus are thin and compliant in the $z$-direction. The mirror surface is coated with aluminum for high reflectivity. The fabrication details are given in the next section.

FEM thermomechanical simulation was conducted using Coventorware [11]. The simulation result is shown in Fig. 2, where the curling of the two sets of bimorph beams compensate each other resulting in a zero initial tilt. The initial elevation of the mirror plate above the chip plane, $Z_{\text {mirror }}$, due to the curling of the thermal actuators can be calculated from:

$$
Z_{\text {mirror }}=\left(L_{\text {frame }}-W_{\text {frame }}\right) \sin \theta
$$

where $L_{\text {frame }}$ and $W_{\text {frame }}$ are the length and beam-width of the frame, respectively. $\theta$, the initial tilt angle of the frame, can be computed from $\theta=l / \rho$; where $l$ and $\rho$ are the length and radius-of-curvature of the thermal actuator, respectively. For a frame with $L_{\text {frame }}=0.5 \mathrm{~mm}, W_{\text {frame }}=40 \mu \mathrm{m}$, and $\theta=17^{\circ}$, the initial rest position of the mirror $Z_{\text {mirror }}$ is $0.135 \mathrm{~mm}$. The simulation results in Fig. 2 show that the mirror plate is located $0.132 \mathrm{~mm}$ above and parallel to the substrate plane. There is no substrate underneath the mirror plate. Thus, the mirror plate can move down below the chip plane. From experiment, it was found that the maximum displacement below the chip plane is roughly equal to $Z_{\text {mirror }}$, thereby resulting in a maximum $z$ displacement, $Z_{\max }=2 Z_{\text {mirror }}=0.27 \mathrm{~mm}$. Much larger vertical displacements can be achieved by simply increasing the frame and/or the actuator lengths.

The mirror and frame actuators rotate the mirror in opposite angular directions. There exist two basic modes of operation: (1) Bi-directional scanning by alternatively applying voltage to the mirror and frame actuators, and (2) Large piston motion by simultaneously applying voltage to both actuators. Equal angular rotation by the two actuators will result in pure vertical (a)

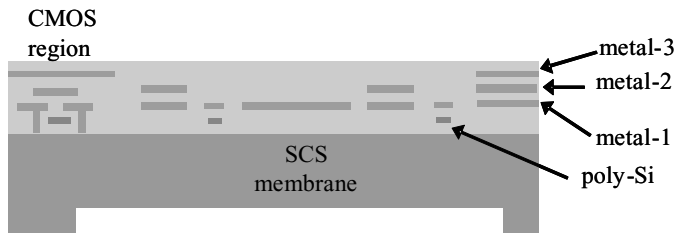

(b)

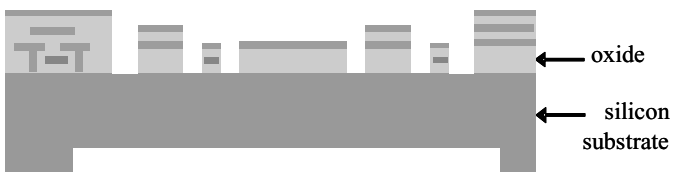

(c)

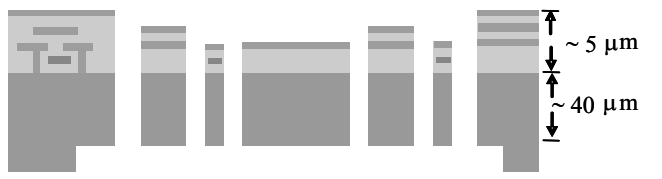

(d)

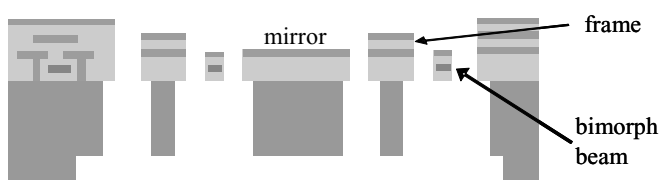

Figure 3. DRIE CMOS-MEMS process flow: (a) backside Si etch; (b) oxide etch; (c) Deep Si etch; and (d) Si undercut.

displacement of the mirror. Large $z$-axis displacement is achieved via the angular amplification due to the long arm length of the frame.

\section{DEVICE FABRICATION}

The micromirror is fabricated using deep-reactive-ion-etch (DRIE) CMOS-MEMS processing [12]. The process flow, outlined in Fig. 3, uses only four dry etch steps and can produce mixed thin-film and bulk-Si microstructures. The process starts with a backside deep anisotropic silicon etch to form a thick SCS membrane $(\sim 40 \mu \mathrm{m})$. This SCS membrane is required to keep the mirror flat. The second step is a frontside anisotropic oxide etch that uses the CMOS interconnect metal (i.e., Al) as an etching mask. Next, a deep silicon trench etch is done to release the microstructure. The last step is an isotropic silicon etch, performed to undercut the silicon to form bimorph thinfilm beams which are approximately $2 \mu \mathrm{m}$ thick. These thinfilm beams provide $z$-axis compliance for out-of-plane actuation, and form bimorph actuators with an embedded polysilicon heater. There are no substrate or other microstructures directly above or below the mirror microstructure, so large actuation range is allowed. This process is mask-less, uses only dry etch steps, and is completely compatible with foundry CMOS processes.

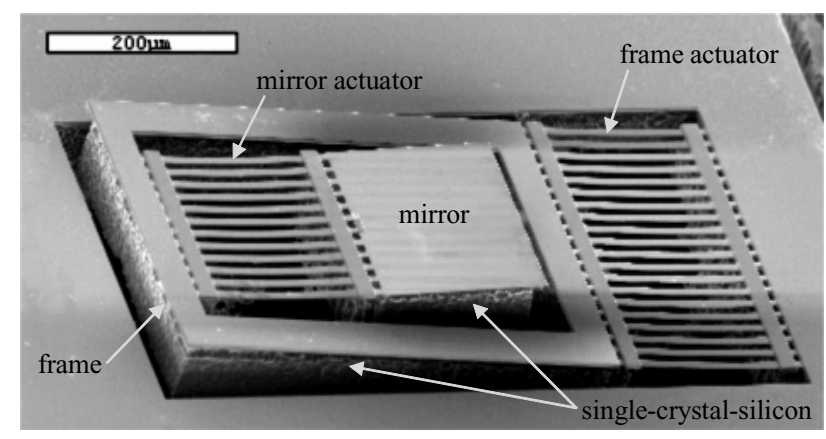

Figure 4. SEM of a fabricated LVD micromirror device. 


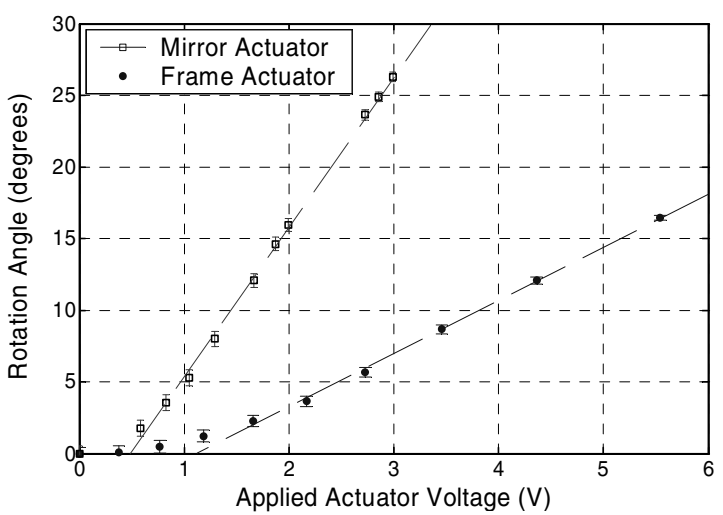

(a)

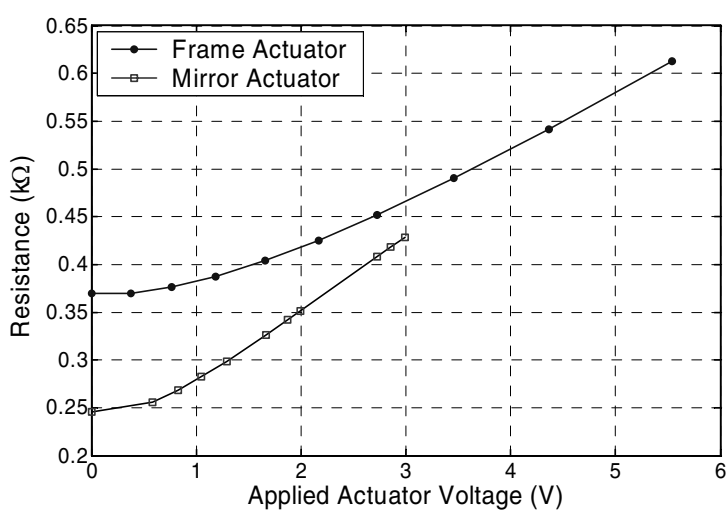

(b)

Figure 5. Device characterization. (a) Plot of the rotation angle, and (b) plot of the polysilicon heater resistance versus applied voltage for the two actuators.

A fabricated $0.7 \mathrm{~mm}$ by $0.32 \mathrm{~mm}$ LVD micromirror device is shown in Fig. 4. The initial tilt angle of the frame with respect to the substrate surface is typically $13^{\circ}$. This tilt angle is dependent on the over-etch of the top aluminum layer of the bimorph beams. The heating element in the $10-\mu \mathrm{m}$ wide bimorph beams is a set of $200 \mu \mathrm{m}$ long, $7 \mu \mathrm{m}$ wide, polysilicon strips oriented along the beams. The gaps $(9 \mu \mathrm{m})$ between the beams are used to undercut the underneath silicon to form the thin-film bimorph beams (Fig. 3(d)). The frame actuator and mirror actuator are constituted of 20 and 12 bimorph beams, respectively. The measured open circuit polysilicon resistances of the mirror and frame actuators are $240 \Omega$ and $365 \Omega$, respectively. The peak-to-valley surface deformations are within $40 \mathrm{~nm}$ over the $190 \mu \mathrm{m}$ mirror plate. This small mirror size is just used to demonstrate the concept. Since the mirror plate is supported by bulk silicon, much larger mirrors can be made.

\section{EXPERIMENTAL RESULTS}

An experimental setup with a laser beam incident on the mirror and a d.c. voltage applied to the two actuators was used to measure the static deflection angles. The mechanical scan angle of the mirror was obtained by measuring the scan distance of this reflected laser beam on a screen. Fig. 5(a) shows the linear responses between the measured angles of rotation and the actuation voltages for the mirror and frame actuators, respectively. The mirror has a maximum rotation of $26.5^{\circ}$ when $3 \mathrm{~V}$ d.c. is applied to the mirror actuator. Both the mirror and the frame rotate when a voltage is applied only to the frame actuator. A maximum deflection of $-16.5^{\circ}$ is observed when $5.5 \mathrm{~V}$ d.c. is applied to the frame actuator. The same actuation voltage causes a larger rotation angle by the mirror actuator than the frame actuator due to the polysilicon resistance difference between the two actuators, and also because the mechanical structure of the frame provides additional thermal isolation to the mirror actuator. The polysilicon resistance of the bimorph actuators change with applied voltage, and is plotted in Fig. 5(b). There is a large increase in the polysilicon resistance because the heating effect of the actuation current causes a temperature change, which in turn induces stress change in the bimorph beams. As evident from Fig. 6, there exists a linear correlation between the rotation angle and the polysilicon resistance for each of the two actuators. The resulting error $(<2 \%$ of full-scale value) in fitting the actuator rotation and resistance data to a linear plot is within the rotation-angle

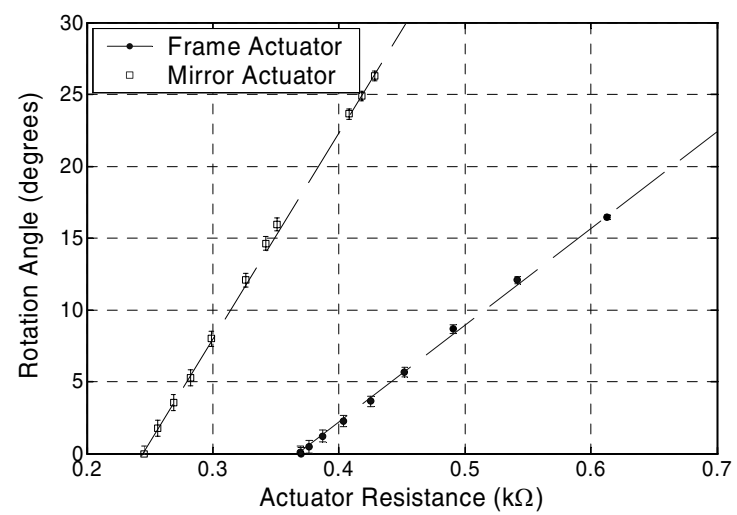

Figure 6. Plot of the linear correlation between rotation angle and the polysilicon resistance for the two actuators.

measurement experimental-error. This linear relationship allows for independent control of rotation angle of each actuator by monitoring its polysilicon heater resistance. Thermal coupling between the two actuators can be accounted for by monitoring their individual polysilicon heater resistances.

Vertical piston motion of the mirror can be achieved by equal but opposite angular rotations of the two actuators. By using the rotation-angle versus actuation-voltage data, a mirroractuator drive-voltage versus frame-actuator drive-voltage plot for same angular rotation values is obtained. The slope of this experimentally determined plot, shown in Fig. 7(a), provides the driving voltage ratio for the two actuators that would maintain no tilting of the mirror plate. A voltage divider was used to drive the two actuators with a voltage ratio of 3:7 (determined from plot). A maximum vertical displacement of $200 \mu \mathrm{m}$ was obtained. The vertical displacement of the mirror as a function of the drive voltage is shown in Fig. 7(b). By using a linearlyfitted voltage ratio, some tilting of the mirror plate (less than $1^{\circ}$ ) was observed.

The frequency response of the LVD micromirror was measured using a Polytec OFV-511 laser Doppler vibrometer, as shown in Fig. 8. Resonant peaks were observed at $1.18 \mathrm{kHz}$ and $2.62 \mathrm{kHz}$. These results are a close match to the modes observed at $1.14 \mathrm{kHz}$ and $2.76 \mathrm{kHz}$ from the modal analysis simulations using Coventorware. 

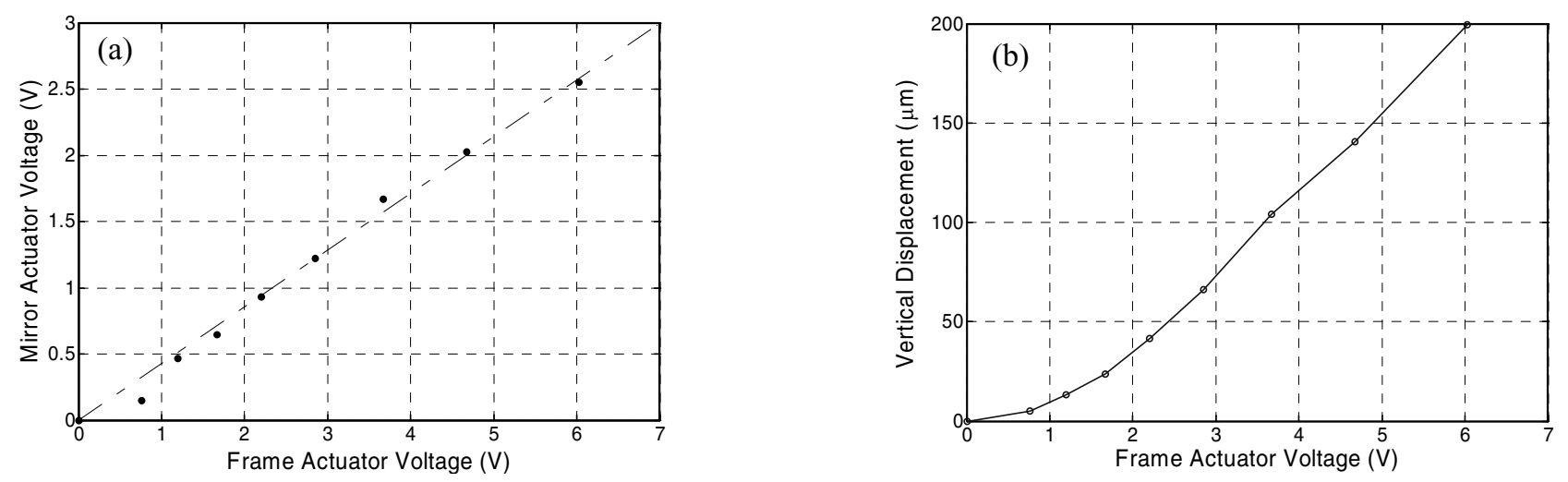

Figure 7. (a)Plot of mirror actuator voltage versus corresponding frame actuator voltage that is required to maintain zero angular tilt of the mirror. (b) Vertical displacement of the mirror as a function of frame actuator voltage. The voltage applied to the mirror actuator is $43 \%$ of the frame actuator voltage, which is provided by a voltage divider. With this voltage ratio the maximum tilt of the mirror in the entire $200 \mu \mathrm{m}$ travel range is less than $1^{\circ}$.

\section{CONCLUSIONS}

A micromirror with a novel large-vertical-displacement electrothermal-actuation mechanism was successfully demonstrated. $200 \mu \mathrm{m}$ vertical displacement has been achieved with a microdevice of only $0.7 \mathrm{~mm}$ by $0.32 \mathrm{~mm}$. Much larger vertical displacement can be achieved by simply increasing the length of the frame and/or the initial tilt angle of the frame. The fabrication process is simple and compatible with CMOS processes; therefore control circuits can be integrated with the mirror on the same chip. This device is capable of large piston motion, and can also operate as a bidirectional scanner with high scanning speed. The large actuation range and fast scanning speeds make this device very suitable for use in interferometry, laser beam steering, biomedical imaging, and phase modulation applications. Based on this LVD microactuator concept, various devices such as tunable microlenses for use in confocal microscopy can also be developed.

\section{REFERENCES}

1. M. A. Helmbrecht, U. Srinivasan, C. Rembe, R. T. Howe, and R. S. Muller, "Micromirrors for Adaptive-Optics Arrays," Technical Digest of the $11^{\text {th }}$ Intl. Conf. on Solid State Sensors and Actuators (Transducers '01), Munich, Germany, June 1014, 2001.

2. A. P. Lee, C. F. McConaghy, G. Sommargren, P. Krulevitch, and E. W. Campbell, "Vertical-Actuated Electrostatic Comb Drive with In Situ Capacitive Position Correction for Application in Phase Shifting Diffraction Interferometry", $J$. of Microelectromechanical Systems, 12, 960-971 (2003)

3. S. $-\mathrm{W}$. Chung, and Y. $-\mathrm{K}$. Kim, "Design and fabrication of $10 \times 10$ micro-spatial light modulator array for phase and amplitude modulation," Sensors and Actuators, 78, 63-70 (1999).

4. S. Kwon, V. Milanovic, and L. P. Lee, "Vertical Microlens Scanner for 3D Imaging", Technical Digest of the 2002 SolidState Sensor and Actuator Workshop, Hilton Head Isl., SC, June 2002, pp. 227-230.

5. D. Lee, U. Krishnamoorthy, K. Yu, and O. Solgaard, "HighResolution, High-Speed Microscanner in Single-Crystalline Silicon Actuated by Self-Aligned Dual-Mode Vertical Electrostatic Combdrive with Capability for Phased Array

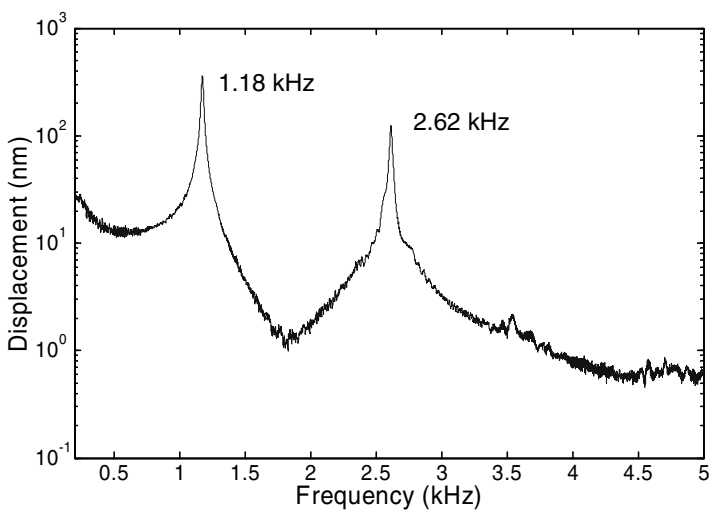

Figure 8. Frequency response of the LVD micromirror device. The actuation signal was a $1 \mathrm{~V}$ sine wave.

Operation", Technical Digest of the $12^{\text {th }}$ International Conference on Solid State Sensors, Actuators and Microsystems (Transducers '03), Boston, MA, June 8-12, 2003, pp. 576-579.

6. O. Cugat, P. Mounaix, S. Basrour, C. Divoux, and G. Reyne, "Deformable magnetic mirror for adaptive optics: first results," IEEE $13^{\text {th }}$ Annual Intl. Conf. on MEMS, Miyazaki, Japan, Jan 2000, pp. 485-490.

7. D. E. Glumac, and W. P. Robbins, "A planar unimorph-based actuator with large vertical displacement capability. I. Experiment," IEEE Transactions on Ultrasonics, Ferroelectrics and Frequency Control, 45, 1145-1150 (1998).

8. A. Tuantranont, L. -A. Liew, V. M. Bright, W. Zhang, and Y. C. Lee, "Phase-only micromirror array fabricated by standard CMOS process," Sensors and Actuators A, 89, 124-134 (2001).

9. H. Xie, A. Jain, T. Xie, Y. Pan, and G.K. Fedder, "A SingleCrystal Silicon-Based Micromirror with Large Scanning Angle for Biomedical Applications," CLEO Technical Digest, Baltimore, MD (2003).

10. A. Jain, T. Xie, Y. Pan, G. K. Fedder, and H. Xie, "A TwoAxis SCS Electrothermal Micromirror for Biomedical Imaging," 2003 IEEE/LEOS International Conference on Optical MEMS, Waikoloa, HI, Aug 2003, pp. 14-15.

11. CoventorWare 2003 Reference Manual, Coventor, Inc., Cary, NC.

12. H. Xie, L. Erdmann, X. Zhu, K. Gabriel, and G.K. Fedder, "Post-CMOS processing for high-aspect-ratio integrated silicon microstructures," J. of Microelectromechanical Systems, 11, 93 101 (2002). 